\title{
BMJ Open FeBRILe3 Project: protocol for a prospective pragmatic, multisite observational study and safety evaluation assessing Fever, Blood cultures and Readiness for discharge in Infants Less than 3 months old
}

Ariel Olivia Mace (D) , ${ }^{1,2,3}$ Andrew C Martin, ${ }^{2,4}$ Jessica Ramsay, ${ }^{3}$ James Totterdell, ${ }^{3}$ Julie A Marsh, ${ }^{3,5}$ Tom Snelling ${ }^{3,6,7,8}$

To cite: Mace A0, Martin AC, Ramsay J, et al. FeBRILe3 Project: protocol for a prospective pragmatic, multisite observational study and safety evaluation assessing Fever, Blood cultures and Readiness for discharge in Infants Less than 3 months old. BMJ Open 2020;10:e035992. doi:10.1136/ bmjopen-2019-035992

- Prepublication history and additional material for this paper are available online. To view these files, please visit the journal online (http://dx.doi. org/10.1136/bmjopen-2019035992).

Received 25 November 2019 Revised 29 February 2020 Accepted 17 April 2020

Check for updates

(c) Author(s) (or their employer(s)) 2020. Re-use permitted under CC BY-NC. No commercial re-use. See rights and permissions. Published by BMJ.

For numbered affiliations see end of article.

Correspondence to

Dr Ariel Olivia Mace;

ariel.mace@health.wa.gov.au

\section{ABSTRACT}

Introduction The purpose of this observational study is to assess the safety and impact of the introduction of a clinical practice guideline $(\mathrm{CPG}$ ) recommending early discharge of infants with fever without source who are at low risk of serious bacterial infection (SBI). We hypothesise that implementation of this guideline will be associated with a rate of unplanned readmission to hospital (within 7 days of discharge) which is similar (ie, non-inferior) to that observed under previous standard practice.

Methods and analysis This observational study is a prospective pragmatic, multisite safety assessment and impact project. It will evaluate the safety of a CPG which allows febrile infants fulfilling low-risk criteria to be discharged early from hospital if their blood cultures demonstrate no growth at 24 hours (compared with previous minimum 48 hours admission). This guideline has been implemented at two Western Australian metropolitan hospitals. Infants aged $<3$ months (chronological or corrected for premature birth before 37 weeks gestation) presenting with fever without source will be included. The primary outcome is readmission to hospital due to clinical deterioration/caregiver concern within 7 days of discharge, identified through review of electronic admission details and study-specific caregiver surveys. Secondary outcomes include rates of $\mathrm{SBI}$, hospital lengths of stay compared with previous practice, clinician guideline adherence and caregiver satisfaction with the discharge process. Analysis will be within a sequential Bayesian safety monitoring framework, which incorporates new information and updates the evidence for guideline safety relative to previous practice (historical control) at prespecified interim analyses. Demographic and clinical information will be summarised.

Ethics and dissemination Ethics approval and waiver of consent for data collection has been granted by the Child and Adolescent Health Service Human Research Ethics Committee (RGS0000001415). Caregivers will have the option to opt out of survey follow-up. Results will be disseminated via peer-reviewed publication.
Strengths and limitations of this study

- This will be the first prospective Australian study to examine the safety of the introduction of a risk stratification protocol for the management of fever without source in infants $<3$ months old.

- This study uses a novel sequential Bayesian safety monitoring framework, which has potential advantages over traditional methods, including updating and incorporation of existing knowledge, straightforward calculation of event probabilities of direct interest, more transparent interpretation of results and the potential to reach an early conclusion if there is clear evidence (based on prespecified criteria) that the policy is safe or unsafe.

- A waiver of consent will ensure the safety cohort are fully representative of the total treatment population meaning that rates of readmission can be validly compared with prior (historical) rates.

- Responses to the electronic (SMS) caregiver survey is voluntary so response rates may be poor.

- By implementing the clinical practice guideline within an active safety monitoring framework, we expect to improve clinician confidence in the change, and thereby facilitate more prompt and complete adoption of the practice.

Trial registration number Australian New Zealand Clinical Trials Registry (ACTRN12619001010189).

\section{INTRODUCTION}

Fever without source (FWS) is one of the most common reasons young infants less than 3 months old present to hospital. FWS is defined as an infant presenting with a fever $>38^{\circ} \mathrm{C}$ (axillary or rectal) without a readily identifiable source on history and/or physical examination (eg, no coryzal or other respiratory signs/symptoms). Most of these infants 
have a self-limiting viral infection $(\sim 90 \%)^{12}$; however, a small number have a serious bacterial infection (SBI), such as urinary tract infection, bacteraemia or meningitis, that requires definitive and timely antibiotic treatment and which must be excluded.

Paediatricians need to balance the risk of failing to identify and adequately treat an SBI against over-investigation and treatment of young infants with benign, self-limiting viral illnesses. Previously, all infants less than 3 months old with FWS were recommended to undergo a series of invasive investigations to exclude SBI (blood culture, urine culture and lumbar puncture), and administered intravenous antibiotics in hospital for a minimum of 48 hours, even when the infant appeared clinically well. ${ }^{3}$ These recommendations were developed to align with laboratory processes, whereby a blood culture could only be reported as 'negative' for bacterial growth after 48 hours of incubation. However, the unnecessary treatment of large numbers of low-risk infants with intravenous antibiotics increases the risk of adverse events and iatrogenic complications, ${ }^{2}{ }^{4}$ and may promote the emergence of antibiotic resistance. Unnecessary hospitalisation results in a burden for families and financial costs for healthcare systems. ${ }^{5}$

Increasing national and international evidence supports a change to this practice. The use of continuous monitoring blood culture systems (CMBCS), as is now standard in most modern laboratories, has allowed for real-time detection and notification of positive blood cultures as opposed to the traditional practice of once daily manual blood culture subculture and review. ${ }^{3}$ CMBCS use means that detection of positive blood cultures nearly always occurs before 24 hours. ${ }^{35}$ Additionally, there has been a decrease in the incidence of bacteraemia in infants and a change in bacterial epidemiology due to conjugate vaccination and maternal intrapartum group B Streptococcus prophylaxis, which has altered the risk:benefit ratio of well-appearing infants remaining hospitalised for 48 hours awaiting blood culture results. ${ }^{6}$ Concurrently, evidence continues to emerge to support the use of risk-stratification protocols which identify low-risk infants who can be safely discharged earlier, or managed without antibiotics or admission at all. ${ }^{17-10}$ A growing body of primary literature suggests that some infants who warrant hospitalisation can be safely discharged at 24-36 hours, ${ }^{127-13}$ and that older infants, with different risk profiles for SBI compared with the younger cohort, may warrant less conservative management. ${ }^{14-16}$

However, there remains a wide variation in testing, treatment and overall resource utilisation in the management of young febrile infants, both in Australia and internationally. ${ }^{15} 17$ There are no nationally accepted guidelines to standardise the care of infants with FWS within Australia. Internationally, quality improvement projects have been implemented to reduce variability in infant sepsis care. ${ }^{13}$ However, implementing widespread change to local practice remains challenging without evidence to demonstrate the safety of earlier discharge in the local population.
Here we describe the clinical protocol for the FeBRILe3 study (Fever, Blood cultures and Readiness for discharge in Infants Less than 3 months old), which will prospectively monitor a safety cohort of infants less than 3 months old admitted with FWS after implementation of a new clinical practice guideline (CPG) allowing for early discharge of well-appearing, low-risk infants. This evidence-based CPG was codeveloped by the general paediatric departments at Perth Children's Hospital (PCH) and Fiona Stanley Hospital (FSH) in Western Australia (WA) to standardise FWS management and has been implemented by general paediatricians across both sites (including authors AOM and ACM). The FeBRILe3 study has been designed to prospectively monitor patient outcomes and safety following the recommended change in practice, rather than rely on retrospective review of outcomes some arbitrary time after implementation. This evaluation will use a sequential Bayesian rate-updating approach using the accumulating data. We aim to validate the risk-stratification algorithm for identifying low-risk patients within our population and assess the safety and impact of the changed guideline on infants, their families and resource utilisation within WA Health.

\section{METHODS}

Design

FeBRILe3 is a prospective pragmatic, single arm, multisite observational study, safety assessment and impact project.

\section{Study setting}

The study is being conducted on the inpatient paediatric wards of PCH and FSH, WA. PCH is a tertiary-level public hospital with 3500 general paediatric admissions per year. FSH is a quaternary hospital with a secondary-level paediatric unit, with 1500 paediatric medical admissions annually. A large local retrospective birth cohort analysis identified that approximately $30 \%$ of hospitalisations within the first 3 months of life are for infection-related diagnoses, with a mean length of stay of 2.4 days (SD 2.3) for infants with a discharge diagnosis of 'viral infection' or 'fever, unspecified'.

\section{Objectives}

The primary objective of FeBRILe3 is to evaluate the safety of the implementation of a new CPG introduced at PCH and FSH that allows infants with FWS who fulfil criteria for being at low risk for SBI to be discharged early from hospital if their blood cultures demonstrate no growth at 24 hours.

The secondary research objectives address gaps in local evidence regarding infections in these infants. These include: (1) describing the epidemiology of local pathogens, both SBI and non-SBI, causing FWS, (2) identifying rates of positive blood cultures and distribution of time to positivity within our cohort, (3) describing the clinical and laboratory characteristics of infants with FWS in our cohort and relationship to SBI and non-SBI diagnoses, (4) 
quantifying the impact of CPG introduction on hospital resource utilisation as measured by hospital length of stay, length of intravenous antibiotic therapy and investigations performed and (5) surveying the self-reported impact of early discharge on families.

\section{Hypothesis}

We hypothesise that the implementation of the new CPG will be associated with a rate of unplanned readmission to hospital within 7 days of discharge which is non-inferior (ie, similar) to that observed under previous standard practice.

\section{Outcomes}

The primary outcome is readmission to hospital within 7 days of discharge due to clinical deterioration/caregiver concern. This outcome will be assessed by: (1) review of electronic admission details for all participants 7-10 days after discharge to monitor for events occurring within 7 days of discharge and (2) surveying the parent/caregiver 7-10 after discharge from hospital to solicit reports of any readmissions within 7 days of discharge.

Secondary outcomes include:

1. Cases of SBI, such as urinary tract infection, bacteraemia and/or bacterial meningitis, confirmed within 7 days of hospitalisation. This outcome will be assessed by identification of all positive microbiological specimens (urine, blood and/or cerebrospinal fluid) through review of electronic patient results for all enrolled patients.

2. Overall hospital length of stay for the index admission. This outcome will be assessed from the date and time of admission and discharge as recorded in the hospitals' patient information systems and calculated on discharge from hospital (completion of hospital admission). Length of stay for readmissions will also be incorporated into the analyses. Length of stay will also be used to assess adherence to CPG recommendations for 'low risk' patient management (anticipated discharge at 24 hours). Documented use of the CPG and reasons for non-adherence will be captured when available.

3. Caregiver satisfaction with discharge process including early discharge (if applicable) ascertained by electronic caregiver survey. This survey has been designed specifically for this study and will be administered by mobile phone SMS linking to an internet-browser based survey 7-10 days post discharge from hospital.

\section{Study duration}

The study is a Bayesian sample-size adaptive design where the study continues until prespecified stopping criteria are met based on evidence from the accumulating data. It is anticipated that it would take up to 24 months to complete the study if 500 participants are required.

\section{Participants}

All infants admitted under the PCH or FSH general paediatric departments will be included if fulfilling the following criteria: (1) aged $\leq 90$ days chronological age for term infants or $\leq 90$ days since 40 weeks gestation if born before 37 weeks gestation and (2) admitted to participating hospital for the primary reason of investigation and management of FWS. Infants not separated from hospital since birth will be excluded.

Dedicated hospital-employed project nurses at both hospitals will attend the daily morning general paediatric handovers to identify eligible infants and will be notified (during weekday office hours) by the treating medical teams of additional eligible infants admitted during the day. Eligible infants admitted on weekends/public holidays will be identified by the senior treating clinician and the project nurse will be notified of these participants the next work day to facilitate study follow-up.

\section{STUDY PROCEDURES}

\section{Safety cohort exposure}

A formal evidence-based CPG has been implemented by general paediatricians at $\mathrm{PCH}$ and FSH (including authors AOM and ACM) for use in routine patient care to standardise the management and risk-stratification of infants admitted for investigation of FWS (online supplementary file 1). This CPG is intended to follow on from the tertiary emergency department guidelines for the initial investigation of infants presenting with FWS (online supplementary file 2); investigations may include but are not limited to full blood count, C-reactive protein (CRP), urine, blood and/or cerebrospinal fluid microscopy and culture, and viral studies, based on clinical presentation, patient age and clinician discretion.

Implementation of this CPG was through a consultative process including: (1) general paediatric and emergency department staff input into CPG creation (including junior medical officers, consultant paediatricians and nursing staff), (2) approval by the hospital Clinical Practice Advisory Committee, (3) dedicated education sessions with general paediatric medical staff prior to and after introduction of the CPG, (4) easy accessibility to the CPG via quick-links on the departmental intranet homepage, (5) regular departmental email reminders with links to the CPG, (6) poster reminders to use the CPG within clinical workrooms on the general paediatric wards.

As per the implemented CPG, infants admitted for investigation of FWS will be eligible for discharge after 24 hours (compared with previous practice of 48 hours) if they fulfil all low-risk criteria and have no growth on blood cultures at 24 hours. Low-risk criteria include: (1) age $\geq 29$ days, (2) absence of pyuria (no white cells in urine, ie, $<10$ per high powered field), (3) CRP $<20 \mathrm{mg} / \mathrm{L}$, (4) absolute neutrophil count $<10 \times 10^{9} / \mathrm{L}$ and white cell count between $4 \times 10^{9} / \mathrm{L}$ and $15 \times 10^{9} / \mathrm{L}$ ), (5) no antibiotics received within 48 hours prior to admission and (6) well appearance on examination. High risk infants (appearing unwell/toxic, $\leq 28$ days old, or with significant medical comorbidities), those who remain clinically unwell or who have investigative tests suggestive of an SBI (eg, urinary tract infection, bacteraemia or bacterial 


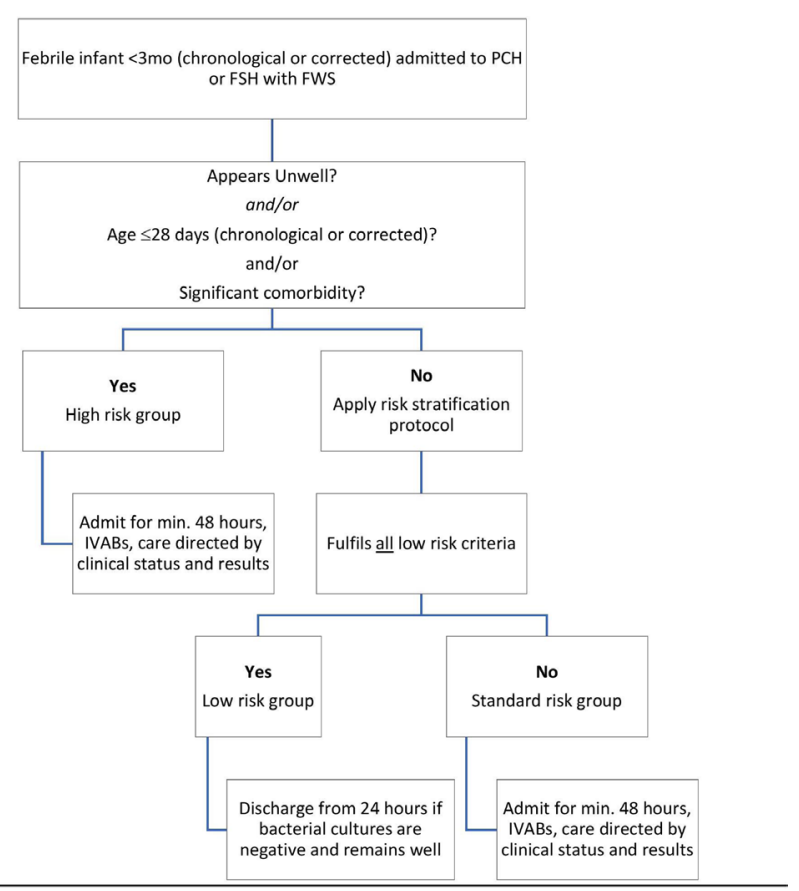

Figure 1 Flow chart directing discharge decision making for well-appearing infants with FWS. FSH, Fiona Stanley Hospital; FWS, fever without source; IVAB, intravenous antibiotics; $\mathrm{PCH}$, Perth Children's Hospital.

meningitis) and infants not fulfilling all low-risk criteria (known as standard risk infants) will be admitted for a minimum of 48 hours, with management directed by clinical status according to established practice (figure 1).

\section{Project specific follow-up}

The safety of a new CPG allowing early discharge of lowrisk infants will be determined by active monitoring of unplanned hospital readmissions rates within 7 days of discharge through review of electronic admission details and caregiver surveys.

A follow-up survey will be sent electronically via SMS (and followed up with a phone call by hospital-employed dedicated project nurses if required) 1 week after discharge (7-10 days) to assess satisfaction with the hospitalisation and discharge process, to ascertain any complications and to identify whether any further medial review was sought since discharge (online supplementary file 3 ).

The observed rate of readmission within 7 days of discharge will be assessed at specified time-points (scheduled analyses) against a preset acceptability threshold for readmissions of $8 \%$; this threshold was derived from a large birth cohort analysis which found the rate of readmissions within 7 days for all infants less than 3 months old with FWS to any WA metropolitan hospital from 2008 to 2012 was $5 \%$, allowing for a $3 \%$ absolute noninferiority margin.

\section{Data collection}

Baseline demographic and clinical information will be collected for all eligible infants. The collection and testing of laboratory specimens will be per routine hospital procedures for FWS investigation (ie, standard of care and clinician discretion). Information for all tests performed, including molecular tests and other investigations for SBI and viral pathogens, will be collected. Clinical variables will be manually extracted from relevant hospital databases and the participant's medical record (containing information on the patient's history including symptoms and signs, the observation chart, results of point-of-care testing if performed and prescribed antibiotic/s), the online emergency department information systems, laboratory information systems and radiology information systems. Demographic, clinical and laboratory information will be summarised and reported. Information on clinical outcome (caregiver report of further fevers, other unplanned medical reviews or hospital admissions within 7 days of discharge) and caregiver satisfaction (very low, low, average, high, very high) with the processes of admission, project information, timeliness of results and review, and discharge, will be obtained by survey administered via electronic communication (email or SMS-linked web survey) or by phone to the parent/caregiver 7-10 days after discharge from hospital.

\section{Waiver of consent for data collection}

As this is a safety initiative, consent will not be requested for the evaluation of routinely collected data of participants within the safety cohort. A waiver of consent for data collection has been granted by the Child and Adolescent Health Service Human Research Ethics Committee (HREC). A waiver of consent will ensure the safety cohort are fully representative of the patient population meaning rates of readmission can be validly compared with prior (historical) rates. Requiring consent could skew the enrolled safety cohort, resulting in either an under or overestimation of the true readmission rate and undermining the validity and scientific value of this project. Inclusion of all infants in the safety cohort will also ensure any safety issues are detected at the earliest possibility, minimising the risk to the patient population.

\section{Opt out consent for project-specific follow-up}

A project-specific survey will be issued to the parents/ caregivers of all infants within the safety cohort. Parents/ caregivers will be informed about the survey and project on both the discharge information sheet provided on hospital discharge and during the telephone follow-up by the hospital treating team within 24 hours of discharge. The written and verbal information provided will allow parents/guardians of participants to make an informed choice about survey participation and they will be able to understand that they can decline to participate ('opt out'). As hospital readmissions rates within 7 days of discharge will primarily be identified through review of electronic admission details, nonparticipation in the follow-up survey is not anticipated to impact on collection of data for the primary safety outcome. 


\section{Sample size}

The study uses sequential Bayesian analyses for monitoring where the trial continues unless prespecified stopping criteria are met (safe/unsafe) based on the accumulating data. A sample size of 500 infants was deemed feasible based on experience at the two sites and the study timeline, anticipating an average of five infants per week. Simulation of the design assuming a maximum sample size of 500 infants estimated $83 \%$ power to declare safety assuming a 7-day readmission rate equal to the historical rate of 0.05 .

\section{Data analysis plan}

The observed rate of readmission within 7 days of discharge will be assessed at specified time-points (scheduled analyses) against the preset acceptability threshold of $0.08(8 \%)$ readmission rate, using a sequential Bayesian safety monitoring framework. ${ }^{18}$ The sequential Bayesian monitoring incorporates new information as the project progresses and updates the current evidence for safety of the guideline at prespecified interim analyses. The project hypothesis is that the 7-day readmission rate under the new guideline, denoted $\theta$, is no worse than the estimated historical rate of 0.05 with an allowed tolerance of 0.03 . In terms of statistical hypotheses, we aim to assess

$$
\begin{aligned}
& H_{0}: \theta \geq 0.08 \\
& H_{1}: \theta<0.08
\end{aligned}
$$

The new 7-day readmission rate, $\theta$, will be estimated using a beta-binomial model, sequentially updated at prespecified interim analyses. The first analysis will take place when follow-up is available on 100 individuals, with each subsequent analysis occurring when follow-up is available on an additional 50 individuals up to the maximum of 500 .

The posterior distribution for the readmission rate at each analysis will be used to make decisions on the safety of the guideline. If

$$
\operatorname{Pr}(\theta<0.08 \mid \text { interm data })<0.05
$$

then further enrolment is ceased for safety reasons. Otherwise enrolment continues until the next analysis. If the maximum sample size is reached, if

$$
\operatorname{Pr}(\theta<0.08 \mid \text { interm data })>0.95
$$

then implementation of the guideline will be declared safe, being no worse than the historical rate plus the maximum tolerable difference. Any other outcome will be declared inconclusive.

\section{Description of sequential Bayesian analysis}

We assume that the historical baseline rate is $\theta_{0}$, and we wish to assess the readmission rate under the new guideline, denoted $\theta$. We declare the new rate acceptable if it is within some clinically determined maximum tolerable difference $\tau$ of the historical rate. Statistically, we aim to assess the following hypotheses:

$$
\begin{gathered}
H_{0}: \theta \geq \theta_{0}+\tau(\text { unsafe }) \\
H_{1}: \theta<\theta_{0}+\tau \text { (safe) }
\end{gathered}
$$

and decide on the safety of the guideline.

We assume analyses are conducted at interims $k=1, \ldots, K-1$ with sample sizes $N_{k}$ and the terminal analysis at $k=K$ at the maximum assumed sample size. We model the number of 7-day readmissions at interim analysis $k$, denoted $y_{k}$, as a binomial random variable governed by the probability of 7-day readmission, $\theta$. For simplicity, we specify a conjugate Beta prior on parameter $\theta$. The complete model is

$$
\begin{gathered}
\pi_{0}(\theta)=\operatorname{Beta}(\theta \mid \alpha, \beta) \\
f_{k}\left(y_{k} \mid \theta\right)=\operatorname{Binomial}\left(N_{k}, \theta\right) \\
\pi_{k}\left(\theta \mid y_{k}\right)=\operatorname{Beta}\left(\theta \mid \alpha+y_{k}, \beta+N_{k}-y_{k}\right) \\
P_{k}=\operatorname{Pr}\left(\theta<\theta_{0}+\tau \mid y_{k}\right)
\end{gathered}
$$

Where $\pi_{k}\left(\theta \mid y_{k}\right)$ is the posterior distribution of the 7-day readmission parameter at analysis $k$, and $P_{k}$ is the posterior probability that the 7-day readmission rate is within the safety bound. The hyperparameters $\alpha$ and $\beta$ will be set to 1 for a non-informative prior distribution on the 7-day readmission parameter.

At each analysis, we decide to stop enrolment for safety concerns if $P_{k}<0.05$, otherwise we allow continued implementation of the guideline. If at the final analysis, $P_{K}>0.95$, then we decide that the 7 -day readmission rate is within the safety bound and declare implementation of the guideline safe. If $P_{K}<0.05$ then we declare implementation of the guideline unsafe. Otherwise, the project is inconclusive with respect to safety.

To estimate the probability of each decision, the expected sample size with SD, expected estimate with $\mathrm{SD}$, under various values of the new readmission rate, $\theta^{\star}$ , Monte Carlo methods were used. For various assumed readmission rates, we ran 10000 simulations from the specified model assuming a first interim analysis when follow-up is available on 100 individuals, and subsequent interim analyses every further 50 individuals with follow-up. The results are summarised in table 1.

Based on these simulations, which assumed a maximum enrolment of 500, we estimated $83 \%$ power to declare safety for a 7-day readmission rate equal to the historical rate of 0.05 . We estimated type I error of approximately $4 \%$ at the boundary of the null hypothesis assuming a 7 -day readmission rate of 0.08 .

\section{Safety monitoring}

Infants will continue to be enrolled into the safety cohort until the practice of early discharge for low-risk infants with early negative blood cultures is demonstrated to be safe (or unsafe). We define evidence of safety (noninferiority to previous practice) as a $>95 \%$ probability that the rate of readmission is $<0.08$ (compared with a previous baseline rate of $\sim 0.05$, allowing for a 0.03 


\begin{tabular}{llllllllll}
\hline Table 1 & FeBRILe3 project operating characteristics \\
\hline $\boldsymbol{\theta}^{\star}$ & $\begin{array}{l}\text { Unsafety } \\
\text { bound }\end{array}$ & $\begin{array}{l}\text { Safety } \\
\text { bound }\end{array}$ & $\mathbb{P}$ (unsafe) & $\mathbb{P}($ safe $)$ & $\mathbb{P}($ stop early) & $\mathbb{E}(\boldsymbol{N})$ & $\mathbb{S}(\boldsymbol{N})$ & $\mathbb{E}(\boldsymbol{\theta})$ & $\mathbb{S}(\boldsymbol{\theta})$ \\
\hline 0.05 & 0.05 & 0.95 & 0.00 & 0.83 & 0.00 & 499 & 18 & 0.05 & 0.01 \\
0.06 & 0.05 & 0.95 & 0.01 & 0.48 & 0.01 & 496 & 39 & 0.06 & 0.01 \\
0.07 & 0.05 & 0.95 & 0.05 & 0.18 & 0.05 & 484 & 74 & 0.07 & 0.02 \\
0.08 & 0.05 & 0.95 & 0.16 & 0.04 & 0.16 & 454 & 115 & 0.09 & 0.02 \\
0.09 & 0.05 & 0.95 & 0.38 & 0.00 & 0.35 & 402 & 150 & 0.10 & 0.02 \\
0.10 & 0.05 & 0.95 & 0.65 & 0.00 & 0.61 & 325 & 164 & 0.11 & 0.02
\end{tabular}

$\theta^{\star}$, new readmission rate; $\mathbb{P}$ (safe), probability that guideline is safe; $\mathbb{P}$ (unsafe), probability that guideline is unsafe; $\mathbb{E}(N)$, expected sample size; $\mathbb{S}(N)$, SD of expected sample size; $\mathbb{E}(\theta)$, expected estimate; $\mathbb{S}(\theta)$, SD of expected estimate.

(absolute) non-inferiority margin); evidence of unsafe practice (inferiority) is defined as a $>95 \%$ probability that the rate of readmission is $\geq 0.08$.

Hospital clinical staff and project nurses will actively monitor for readmissions (primary endpoint) by: (1) review of the electronic admission details for all participants 7-10 days after discharge to monitor for events occurring within 7 days of discharge and (2) surveying the parent/caregiver 7-10 after discharge from hospital to ask directly about readmissions. All readmissions will be reviewed by a safety review group (SRG), comprising doctors with expertise in clinical paediatrics. The aim of the SRG is to: (1) determine the reason for readmission, (2) monitor for safety events indicating serious morbidity (eg, paediatric intensive care unit (PICU) admissions) or mortality and to (3) provide clinical opinion regarding external factors that may affect statistical modelling (eg, an outbreak of influenza affecting hospital readmission rates, or unexpected high rates of contaminated specimens requiring readmission for repeat testing). Formal analysis of the primary safety endpoint will be done in accordance with the statistical analysis plan. Other safety events occurring during the patient's admission, defined as a clinical incident that has or could have caused moderate harm, serious harm or death, will be identified and documented by the project nurses during case-note review and will be reported to the SRG. Clinical incidents not pertaining to the primary endpoint will be managed as per hospital policy and will be reported in the study findings on completion of the study.

\section{Statistical monitoring}

An Independent Statistical Monitoring Committee (ISMC) will provide independent statistical oversight of adherence to the predefined project decision making processes for the study, through the monitoring of: (1) the quality of the statistical analyses; (2) the implementation of project adaptations if statistical triggers are met and (3) any threats to the integrity of the ongoing project that arise from inappropriate communication of interim results to the investigators. The ISMC will receive and review updates of the Bayesian analyses and appropriate summaries from the project biostatistician after each scheduled interim.

\section{Potential benefits and risks to participants}

Expected benefits of this project include improved safety of hospital management for febrile infants and facilitation of more prompt and complete adoption of the recommended practice. We expect our experience will provide evidence to translate the practice to other secondary and tertiary paediatric health services.

Studies examining hospital length of stay for common paediatric presentations have not found an association between shorter lengths of stay and increased readmission rates ${ }^{19} 20$; therefore, we anticipate that early discharge for low risk infants will not be associated with an increased risk of hospital readmission. We will also be actively monitoring for serious safety outcomes indicating serious morbidity (eg, PICU admissions) or mortality.

Potential risks, although unlikely, relate to breaches of confidentiality/disclosure of individual identity. Participant privacy will be ensured by de-identifying data at the earliest opportunity and allocation of a participant project number. De-identified participant data will be recorded on a secure, password-protected project database. A list of participant project numbers linked to participant will be stored as a password-protected document and kept on the site principal investigator's (PI) networked health computer, accessed only by the site PI to allow re-identification if necessary. Only the project team members will have access to password-protected information on the secure project database.

\section{Patient and public involvement}

FeBRILe3 has received endorsement from the Wesfarmers Infectious Disease Community Reference Group (IDCRG). The IDCRG were supportive of the premise of this project and its value in improving and assessing the safety of healthcare for the infant population. The IDCRG provided feedback regarding the project design, the legitimacy of waiver of consent and the follow-up survey questions and administration method. IDCRG 
reviewed and endorsed the plain language summary and follow-up questionnaire.

\section{DISCUSSION}

Although policies of early discharge of well-appearing infants have been implemented in a number of settings, no Australian studies have prospectively evaluated the safety of such policy or guideline. FeBRILe3 aims to determine the safety of early discharge for low-risk infants by active monitoring of unplanned hospital readmissions rates within 7 days of discharge. Unplanned readmission rates have been used previously to monitor quality of patient care and to drive quality healthcare improvement. ${ }^{21}$ Participants will be actively monitored to confirm the safety of early discharge in the first 500 participants managed under the guideline; conversely, if readmission rates are found to be unacceptably higher than the historic rate using continuous Bayesian rate-updating, the early discharge guideline will be suspended pending further investigation. Sequential Bayesian safety monitoring has potential advantages over traditional methods, such as updating/incorporation of existing knowledge, straightforward calculation of event probabilities of direct interest, more transparent interpretation of results and the potential to reach an early conclusion if the safety or unsafety are clear. For monitoring, at each analysis the posterior probability of the parameter of interest is computed from the prior probability and the interim data. The current evidence for safety captured in the posterior distribution can be used to inform decisions on whether to continue with or stop implementation of the guideline.

Our approach requires the collection of demographic and baseline data for a safety cohort of all infants aged $<3$ months admitted with FWS to both PCH and FSH. There is a paucity of local research into optimal management of this cohort. The data collected through FeBRILe3 will provide the opportunity to build a multisite patient database to address further clinical questions about the local management of these infants. Future questions include identifying which infants may be safely managed without specific investigations (including lumbar punctures), without antibiotics or without admission at all.

There are no anticipated costs associated with early discharge, numerous potential benefits and, we expect, acceptable risk. Patients and families may benefit from spending less time in hospital with fewer iatrogenic complications associated with hospitalisation, and the health service could benefit from the improved bed capacity from the shorter length of stay. Earlier discharge of suitable patients will reduce hospital stays and help reduce patient caseloads for clinical staff. Consequentially, implementing change to standard practice in the management of febrile infants, particularly reducing length of stay without affecting patient outcomes, could have a substantial positive impact on health services. By implementing the guideline within an active safety monitoring framework, we expect to improve clinician confidence in the change, and thereby facilitate more prompt and complete adoption of the practice. We expect our experience will provide evidence to translate the practice to other paediatric health service providers across Australia and beyond.

\section{ETHICS AND DISSEMINATION}

The study team will ensure that this project is conducted in accordance with the principles of the Declaration of Helsinki. The study team will ensure that this project is conducted in full conformity with relevant regulations and with the International Conference on Harmonisation Guidelines for Good Clinical Practice (CPMP/ ICH/135/95) July 1996.

Ethics approval has been granted by the Child and Adolescent Health Service HREC (RGS0000001415). Planned deviations from this protocol will not occur without approval from the relevant governing bodies.

Dissemination of results will occur through peerreviewed publications and presentation(s) to important stakeholders.

\section{Author affiliations}

${ }^{1}$ Department of Paediatrics, Fiona Stanley Hospital, Murdoch, Western Australia, Australia

${ }^{2}$ Department of General Paediatrics, Perth Children's Hospital, Nedlands, Western Australia, Australia

${ }^{3}$ Wesfarmer's Centre of Vaccines and Infectious Diseases, Telethon Kids Institute, The University of Western Australia, Nedlands, Western Australia, Australia

${ }^{4}$ School of Medicine, The University of Western Australia, Perth, Western Australia, Australia

${ }^{5}$ School of Mathematics \& Statistics, The University of Western Australia, Perth, Western Australia, Australia

${ }^{6}$ Department of Infectious Diseases, Perth Children's Hospital, Nedlands, Western Australia, Australia

${ }^{7}$ Menzies School of Health Research, Charles Darwin University, Darwin, Northern Territory, Australia

${ }^{8}$ Curtin University, Perth, Western Australia, Australia

Acknowledgements The authors acknowledge the contribution of the Independent Statistical Monitoring Committee (Dr Todd Graves and A/Professor James McGree).

Contributors AOM, ACM, JAM and TS conceptualised the study, wrote the grant proposal and obtained funding. AOM, ACM, JAM and TS designed the details of the study, with substantial contributions from JR and JT. AOM is the responsible primary investigator of the project. AOM prepared the first draft of the manuscript. All authors contributed to critically revising the manuscript for important intellectual content, gave final approval of the version to be published and agree to be accountable for the work as guarantors. The corresponding author attests that all listed authors meet authorship criteria and that no others meeting the criteria have been omitted.

Funding This study is funded by a Western Australia Health Research Translation Project 2018 grant 'FeBRILe3-Fever, Blood cultures and Readiness for discharge in Infants Less than 3 months old'. Author AOM is supported by a Western Australia Health Translation Network Early Career Fellowship, the Australian Government's Medical Research Future Fund (MRFF) as part of the Rapid Applied Research Translation Project, and an Australian Government Research Training Programme Fees Offset. Author TS is supported by a Career Development Fellowship from the National Health and Medical Research Council (GNT1111657).

Competing interests None declared. 
Patient and public involvement Patients and/or the public were involved in the design, or conduct, or reporting, or dissemination plans of this research. Refer to the Methods section for further details.

Patient consent for publication Not required.

Provenance and peer review Not commissioned; externally peer reviewed.

Open access This is an open access article distributed in accordance with the Creative Commons Attribution Non Commercial (CC BY-NC 4.0) license, which permits others to distribute, remix, adapt, build upon this work non-commercially, and license their derivative works on different terms, provided the original work is properly cited, appropriate credit is given, any changes made indicated, and the use is non-commercial. See: http://creativecommons.org/licenses/by-nc/4.0/.

ORCID iD

Ariel Olivia Mace http://orcid.org/0000-0002-8621-5664

\section{REFERENCES}

1 Dorney K, Bachur RG. Febrile infant update. Curr Opin Pediatr 2017;29:280-5.

2 Biondi EA, Byington CL. Evaluation and management of febrile, Wellappearing young infants. Infect Dis Clin North Am 2015;29:575-85.

3 Lefebvre CE, Renaud C, Chartrand C. Time to positivity of blood cultures in infants 0 to 90 days old presenting to the emergency department: is 36 hours enough? J Pediatric Infect Dis Soc 2017;6:28-32.

4 Mintegi S, Gomez B, Martinez-Virumbrales L, et al. Outpatient management of selected young febrile infants without antibiotics. Arch Dis Child 2017;102:244-9.

5 Biondi EA, Mischler M, Jerardi KE, et al. Blood culture time to positivity in febrile infants with bacteremia. JAMA Pediatr 2014;168:844-9.

6 Garber MD. Earlier discharge of febrile infants. AAP Grand Rounds 2014;32:50

7 Vos-Kerkhof Ede, Gomez B, Milcent K, et al. Clinical prediction models for young febrile infants at the emergency department: an international validation study. Arch Dis Child 2018;103:1033-41.

8 Gomez B, Mintegi S, Bressan S, et al. Validation of the "Stepby-Step" Approach in the Management of Young Febrile Infants. Pediatrics 2016;138:e20154381.
9 Irwin AD, Wickenden J, Le Doare K, et al. Supporting decisions to increase the safe discharge of children with febrile illness from the emergency department: a systematic review and meta-analysis. Arch Dis Child 2016:101:259-66.

10 Huppler AR, Eickhoff JC, Wald ER. Performance of low-risk criteria in the evaluation of young infants with fever: review of the literature. Pediatrics 2010;125:228-33.

11 Mintegi S, Bressan S, Gomez B, et al. Accuracy of a sequential approach to identify young febrile infants at low risk for invasive bacterial infection. Emerg Med J 2014;31:e19-24.

12 Kuppermann N, Dayan PS, Levine DA, et al. A clinical prediction rule to identify febrile infants 60 days and younger at low risk for serious bacterial infections. JAMA Pediatr 2019;173:342-51.

13 Biondi EA, McCulloh R, Staggs VS, et al. Reducing variability in the infant sepsis evaluation (revise): a national quality initiative. Pediatrics 2019;144. doi:10.1542/peds.2018-2201. [Epub ahead of print: 21 Aug 2019].

14 Biondi EA, Lee B, Ralston SL, et al. Prevalence of bacteremia and bacterial meningitis in febrile neonates and infants in the second month of life: a systematic review and meta-analysis. JAMA Netw Open 2019;2:e190874.

15 Aronson PL, Thurm C, Alpern ER, et al. Variation in care of the febrile young infant $<90$ days in US pediatric emergency departments. Pediatrics 2014;134:667-77.

16 , Roberts KB, Subcommittee on Urinary Tract Infection, Steering Committee on Quality Improvement and Management. Urinary tract infection: clinical practice guideline for the diagnosis and management of the initial UTI in febrile infants and children 2 to 24 months. Pediatrics 2011;128:595-610.

17 Aldridge P, Rao A, Sethumadavan R, et al. Fever under 3 months and the full septic screen: time to think again? A retrospective cohort study at a tertiary-level paediatric Hospital. $J$ Paediatr Child Health 2018;54:272-8.

18 Thall PF, Simon RM, Estey EH. Bayesian sequential monitoring designs for single-arm clinical trials with multiple outcomes. Stat Med 1995;14:357-79.

19 Gay JC, Hall M, Markham JL, et al. Association of extending Hospital length of stay with reduced pediatric hospital readmissions. JAMA Pediatr 2019;173:186.

20 Morse RB, Hall M, Fieldston ES, et al. Children's hospitals with shorter lengths of stay do not have higher readmission rates. $J$ Pediatr 2013;163:e1031:1034-8.

21 Nakamura MM, Toomey SL, Zaslavsky AM, et al. Measuring pediatric hospital readmission rates to drive quality improvement. Acad Pediatr 2014;14:S39-46. 